\title{
Emergency department HIV testing: engagement in care after diagnosis
}

K. Quinn ${ }^{1}$, K. Flanagan ${ }^{1}$, C. Russell1 ${ }^{1}$, H. Alexander ${ }^{2}$

${ }^{1}$ King's College Hospital NHS Foundation Trust, London, UK. '2Guy's \& St Thomas's NHS Foundation Trust, London, UK

King's College Hospital WTS

Background

NICE recommend expanded HIV testing in areas of very high prevalence (>5 per 1000) including Emergency Departments (EDs). The ED at King's College Hospital serves the boroughs of Lambeth and Southwark, ranked as $1^{\text {st }}$ and $2^{\text {nd }}$ for HIV prevalence in the UK ${ }^{1}$. Opt-out HIV testing was introduced in the ED on 08.08 .2016 for patients $\geq 18$ years who were having a blood test done for another reason. We report upon the first 52 weeks of the project including absolute number of diagnoses and HIV prevalence rate within the ED. Demographics and linkage to care 6 months after identification were analysed for newly diagnosed patients and those who had been disengaged from care for $>12$ months.

Methods

Data were collated for the 52 week testing period (08.08.2016-08.08.2017). HIV positive patients identified through the project were categorised as newly diagnosed, disengaged from care (not seen in a HIV service for $>12$ months before their ED test) or engaged in care. A case note review was undertaken for those who were newly diagnosed and those who had previously disengaged from care 6 months after each person's ED test to determine whether they were: 1) Linked to care, on ART 2) Linked to care, not on ART 3) Not linked to care or 4) Deceased. Patient demographics including age and ethnicity were collected for all those newly diagnosed and those who had disengaged from care.

Results

Over the 52 week period, there were 44,091 unique patient episodes where blood tests were taken. HIV tests were performed in 25,852 episodes equating to a testing rate of $58.6 \%$. Excluding patients who were tested more than once, 190 HIV positive individuals were identified giving a prevalence of 7.3 patients per 1000 within the ED. $16.8 \%$ (32/190) were new diagnoses, $8.9 \%(17 / 190)$ had disengaged from care and $68.9 \%(131 / 190)$ were confirmed to be in care. A further $5.3 \%$ $(10 / 190)$ could not be contacted and therefore it could not be established if they were in care at another centre. $68.9 \%$ $(131 / 190)$ of those with positive tests resided in the two local boroughs.

Of the 32 new diagnoses, $28 \%$ (9/32) were female and $72 \%$ (23/32) male. Black African represented the largest single ethnic group $(47 \%, 15 / 32)$.

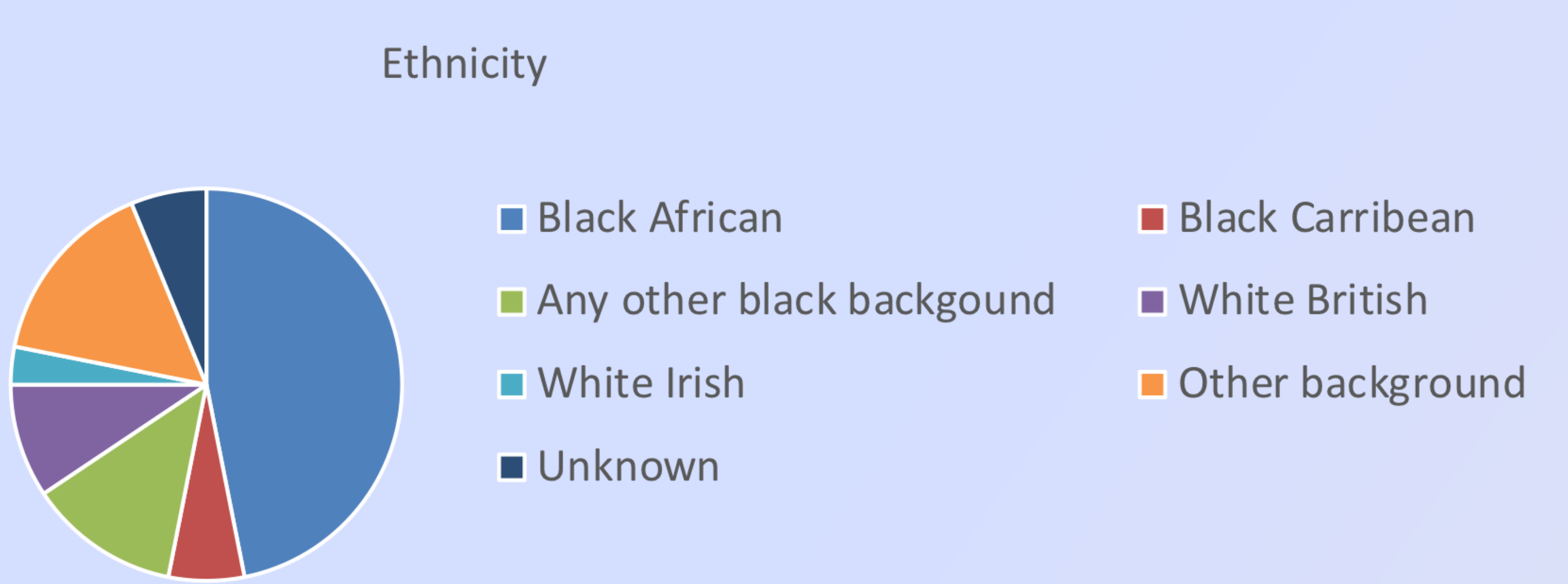

The age range for those with a new diagnosis of HIV was 21-61 and for those previously lost to follow up was 24-60 years old.
$62.5 \%(20 / 32)$ newly diagnosed patients were late diagnoses (CD4 count $<350$ cells $/ \mu \mathrm{L}$ ) and $50 \%(16 / 32)$ were very late diagnoses (CD4 count $<200$ cells $/ \mu \mathrm{L}$ ).

For patients who had disengaged from care and who were reidentified through ED, only $47 \%$ are now linked with care as a result of ED testing.

6-month linkage with care results for newly diagnosed individuals and those who had previously disengaged from care are summarised below:

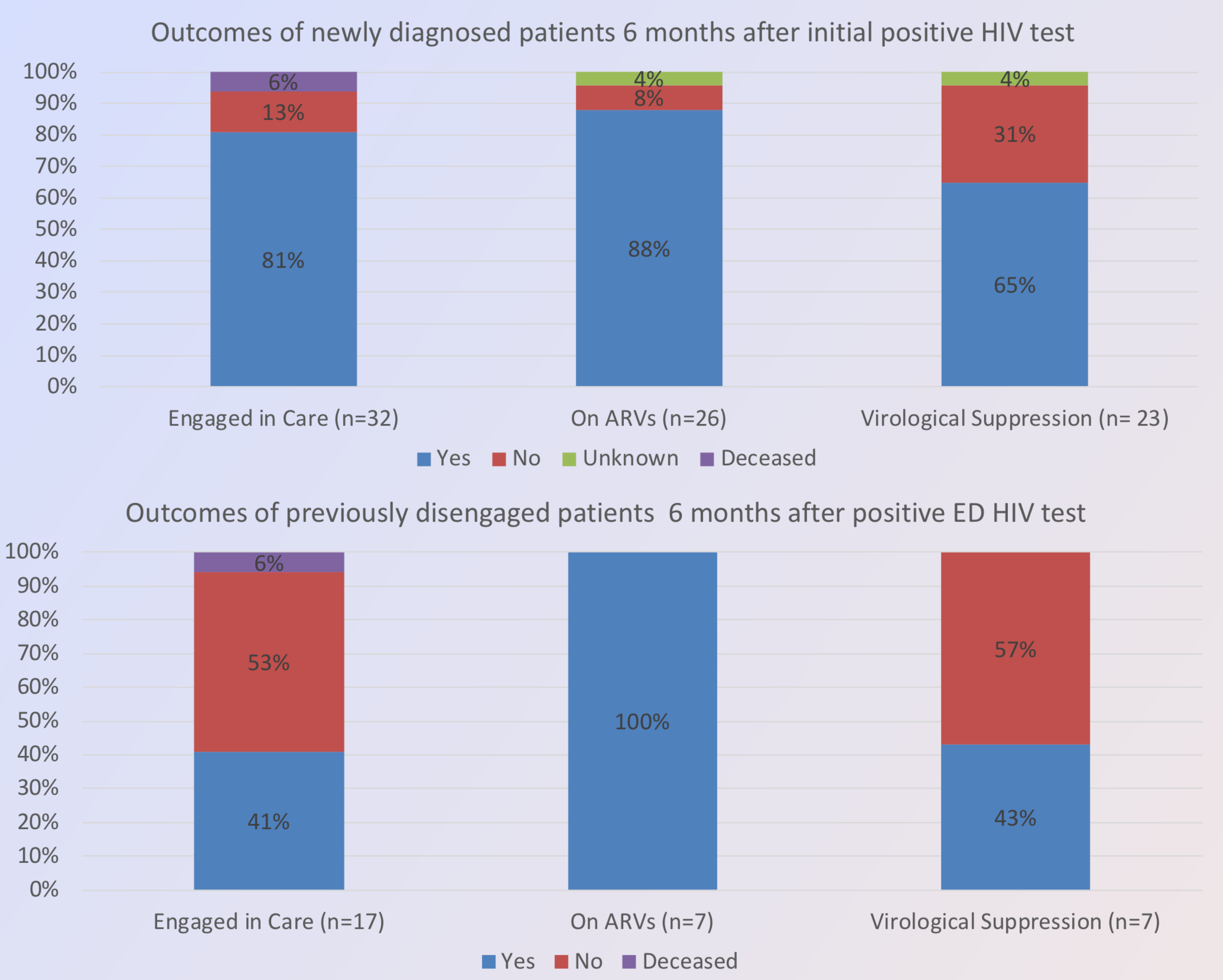

\section{Conclusions}

In an area of very high prevalence, a high uptake of HIV testing has been achieved through ED testing. There was a 6 month mortality rate of $6 \%$ in those who were newly diagnosed and those who had disengaged from care, reflecting that patients identified through ED tend to be late presenters and thus are more unwell. For patients who had disengaged from care and who were re-identified through ED testing, linkage to care remains poor at only $47 \%$ at 6 months. The proportion of PLWHIV with undiagnosed infections was higher than national estimates within 2017 Public Health England dataset (16.8\% vs. 11.6\%) although, this may be because patients who knew they were HIV positive already opted-out of testing ${ }^{2}$. The HIV prevalence within the ED was lower than that of the local population (7.3 per 1000 in ED, 11.7 per 1000 in Southwark, 16.1 per 1000 in Lambeth) $)^{1}$. This may partially be explained by the fact that ED has a higher proportion of older patients attending. Analysis of the differences between those who had an HIV test compared to those who did not is planned to identify reasons for the lower than expected ED prevalence. As HIV incidence decreases and the undiagnosed HIV prevalence fall, targeted approaches to ED testing will become necessary to maintain cost effectiveness.

References
1. Annual epidemiological spotlight on HIV in London: 2016 data. Public Health England, 2016. Available at: https://www.gov.uk/government/publications/hiv-london-annual-data-spotlight
2. Towards elimination of HIV transmission, AIDS and HIV-related deaths in the UK, Public Health England 2017. Available at:
https://assets.publishing.service.gov.uk/government//uploads/system/uploads/attachment_data/file/675809/Towards_elimination_of_HIV_transmission_AIDS_and_HIV_related_deaths_in_the_UK.pdf 